\title{
Características clínicas e epidemiológicas dos partos ocorridos em um hospital maternidade da cidade de Sobral/CE
}

\author{
Clinical and epidemiological characteristics of delivery in a maternity \\ hospital in the city of Sobral/CE
}

\begin{abstract}
Amanda Cavalcante Moreira ${ }^{1}$, Victória de Maria Pereira Rocha Santos ${ }^{1}$, Sarah Gonzalez de Andrade ${ }^{2}$, Yuri Ananias de Vasconcelos ${ }^{1}$, Samaia de Sousa Bem ${ }^{1}$, Rebecca Queiroz de Castro', José Klauber Roger Carneiro ${ }^{3}$, Maria Auxiliadora Silva Oliveira ${ }^{4}$
\end{abstract}

\begin{abstract}
Moreira AC, Santos VMPR, Andrade SG, Vasconcelos YA, Bem SS, Castro RQ, Carneiro JKR, Oliveira MAS. Características clínicas e epidemiológicas dos partos ocorridos em um hospital maternidade da cidade de Sobral/CE / Clinical and epidemiological characteristics of delivery in a maternity hospital in the city of Sobral/CE. Rev Med (São Paulo). 2018 nov.-dez.;97(6):554-60.
\end{abstract}

RESUMO: Objetivos: Objetivou-se traçar as características clínicas e epidemiológicas de parturientes assistidas em um hospital e maternidade do interior do Ceará. Metodologia: Tratase de uma pesquisa retrospectiva, quantitativa e descritiva, tendo como fonte de dados os prontuários de gestantes atendidas no ano de 2015. Foi analisado um total de 1.111 prontuários sendo coletado o tipo de parto, característica dos partos normais, índice de Apgar aos 5 minutos, idade materna, número de consulta prénatal, idade gestacional, escolaridade e justificativa para o parto cesário. Resultados: Os resultados revelam que o parto cesário foi predominante com $50,67 \%$. Em relacão às características dos partos normais houve $35,42 \%$ de episiotomia. Tanto no parto vaginal quanto cesário, tiveram índice de Apgar aos 5, entre 8 e 10 em sua maioria; houve predomínio da idade materna entre 21-30 anos de idade; em relação ao número de consultas foram realizadas mais de 7 consultas; para duração de gestação a maioria esteve entre 37 e 41 semanas, sendo considerada a termo; mães com nível médio de escolaridade fizeram mais parto cesário e $27,27 \%$ destes partos foram justificados por altorisco/pré - eclampsia. Conclusão: Há necessidade por parte do poder público investir mais em educação sexual, visto que foi encontrado gestante em idade menor. Também em relação ao número alto de partos cesarianos há que se ter maior atenção sobre o assunto, já que encontra-se fora dos padrões numéricos estabelecidos.

Descritores: Idade materna; Parto; Cuidado pré-natal; Escolaridade; Idade gestacional.

\begin{abstract}
Objectives: The aim was to trace the clinical and epidemiological characteristics of assisted pregnant women at a hospital and maternity ward in the interior of Ceará. Methods: This was a retrospective, quantitative and descriptive study, having as a data source the medical records of pregnant women attended in the year 2015. A total of 1.111 records were analyzed, and the type of delivery, characteristics of vaginal deliveries, Apgar score at 5 minutes, maternal age, number of prenatal visits, gestational age, schooling, and justification for cesarean delivery were analyzed. Results: The results show that the cesarean delivery was predominant at $50.67 \%$. Regarding the characteristics of vaginal deliveries, episiotomy was found to be $35.42 \%$. Both vaginal and cesarean deliveries had an Apgar score of 5' mostly between 8 and 10; there was a predominance of maternal age between 21-30 years; in relation to the number of prenatal visits made, there were more than 7 visits; for duration of pregnancy, the majority was between 37 and 41 weeks, being considered at term; mothers with secondary schooling had more cesarean deliveries, and $27.27 \%$ of these deliveries were justified by highrisk/preeclampsia. Conclusion: There is a need on the part of the public power to invest more in sex education, since it was found pregnant at a younger ages. Also in relation to the high number of cesarean deliveries, greater attention on the issue is necessary, since it is above the established numerical standards.
\end{abstract}

Keywords: Maternal age; Parturition; Prenatal care; Educational status; Gestational age.

1. Acadêmica(o) do curso de Medicina da Centro Universitário Inta - UNINTA, membro da Liga Acadêmica de Embriologia Integrada a Histologia LAEH. ORCID: Moreira AC - https://orcid.org/0000-0001-7985-3329; Santos VMPR - https://orcid.org/0000-0003-4282-8183; Vasconcelos YAV https://orcid.org/ 0000-0003-4419-599X; Bem SS - https://orcid.org/0000-0001-8822-2342; Castro RQ - https://orcid.org/0000-0001-7114-7067. Email: amandacm@hotmail.com, vick rocha@hotmail.com, yurinanias vasconcelos@hotmail.com, samaia souza@hotmail.com, rebeccaqc@hotmail.com.

2. Acadêmica do curso de Medicina do Centro Universitário Christus - Unichristus. ORCID:https://orcid.org/0000-0003-1031-4487. Email: gonzalezsarah27@gmail.com.

3. Docente do curso de Medicina da Centro Universitário Inta-UNINTA. ORCID: https://orcid.org/0000-0001-8849-5498. Email: ecobio@zipmail.com.

4. Docente do curso de Medicina da Centro Universitário Inta - UNINTA, membro da Liga Acadêmica de Embriologia Integrada a Histologia - LAEH. ORCID: https://orcid.org/0000-0002-2850-146X. Email: myresearchbio@hotmail.com.

Endereço para correspondência: Amanda Cavalcante Moreira. Centro Universitário Inta - UNINTA. R. Cel. Antonio Rodrigues Magalhães, 359.

Bairro: Dom Expedito Lopes, Sobral, CE. CEP: 62050-100. Email: amandacm@hotmail.com 


\section{INTRODUÇÃO}

Assistência pré-natal é um importante elemento Ada atenção à saúde materno-infantil, pois permite a identificação de fatores de risco para a gravidez e possibilita seu gerenciamento ${ }^{1}$ O acompanhamento apropriado do pré-natal está associado a melhores resoluções perinatais ${ }^{1} \mathrm{e}$ à redução da mortalidade materna e infantil ${ }^{2}$.

No Brasil, a atenção pré-natal segue as recomendações do Ministério da $\mathrm{Saúde}^{3}$ e tem como objetivo diminuir a mortalidade materno-infantil no país. Segundo o Ministério da Saúde, a atenção pré-natal deve ser acolhedora, sem interferências desnecessárias, iniciada durante o primeiro trimestre de gestação e com a realização de, no mínimo, seis consultas de pré-natal (ao menos uma consulta no primeiro trimestre, duas no segundo trimestre e três no terceiro trimestre de gestação). Tais recomendações têm o objetivo de favorecer as condições de atendimento às gestantes na rede pública de saúde, como forma de minimizar a mortalidade materna e perinatal ${ }^{3,4}$.

A gravidez, o parto e o nascimento, além de serem inspirados pela organização e pelas práticas dos serviços de saúde, agregam-se a fatores socioeconômicos e demográficos, tais como: escolaridade, trabalho, renda, situação conjugal, idade e raça ${ }^{5-8}$. Nesse cenário, as políticas sociais brasileiras, entre elas a da saúde, apresentam como característica relevante o favorecimento dos grupos sociais mais apaniguados em detrimento dos segmentos de maior vulnerabilidade social ${ }^{9}$, de modo que as desigualdades em saúde entre grupos e indivíduos representam uns dos traços mais marcantes da situação da saúde do Brasil ${ }^{10}$.

Tendo em vista a contribuir para o conhecimento da realidade das características clínicas e epidemiológicas dos partos, a fim de subsidiar trabalhos na melhoria da saúde pública, o presente trabalho objetivou traçar as características clínicas e epidemiológicas dos partos ocorridos em um hospital e maternidade da cidade de Sobral/CE.

\section{METODOLOGIA}

Trata-se de um estudo de caráter exploratório, quantitativo, retrospectivo, com análise documental. A pesquisa foi realizada em um hospital e maternidade situado no município de Sobral/CE. O referido hospital é referência regional e estadual, em atendimento de saúde de alta complexidade, com mais de 90 anos. As unidades hospitalares hoje atendem juntas cerca de 40 mil pacientes por mês e contribuem para formação de acadêmicos de áreas diversas, consolidando-se também como um hospital de ensino. Durante seu dia-a-dia, o hospital tem por finalidade promover assistência, ensino, pesquisa e extensão, prestando serviços de saúde com qualidade, através de uma assistência humanizada e da formação de profissionais da área, visando à satisfação de seus colaboradores e usuários. Ser reconhecida como uma instituição de excelência em gestão e prestação de serviço de saúde, primando pela assistência, ensino e pesquisa é sua meta ${ }^{11}$.

Os sujeitos da pesquisa foram as parturientes $(\mathrm{n}=1.111)$ atendidas no referido hospital, cujos prontuários datassem do ano de 2015. Foram excluídas as fichas e/ou prontuários de anos diferentes deste tempo préestabelecido.

As variáveis analisadas foram aquelas que permitissem traçar característica clínicas e epidemiológicas tais como: tipo de parto, característica dos partos normais, índice de Apgar aos 5 minutos, idade materna, número de consulta pré-natal, idade gestacional, escolaridade e indicação para a realização do parto cesário.

Os dados foram coletados a partir dos prontuários de acompanhamento das parturientes que foram atendidas no hospital. Anexado a este estava a Declaração de Nascidos Vivos, que foi fonte de coleta de muitas informações que permitissem traçar o perfil. Os dados foram analisados em Microsoft Excell e foram confeccionadas tabelas contendo frequências absolutas e relativas.

O presente trabalho foi submetido ao comitê de ética da Universidade Estadual do Vale do Acaraú (1.402.425) e manteve o anonimato e seguiu as recomendações da Portaria do Conselho Nacional de Saúde/MS - CNS, Resolução 466/12, adotando os quatro princípios básicos da bioética: autonomia, beneficência, não maleficência e justiça.

\section{RESULTADOS}

Na Tabela 1 estão os resultados referentes aos tipos de partos realizados nas gestantes atendidas. Como pode ser observado o parto do tipo cesário foi o predominante.

Tabela 1 - Tipo de parto em parturientes atendidas em um hospital e maternidade da cidade de Sobral/CE

\begin{tabular}{lcc}
\hline Tipo de parto & $\mathbf{n}$ & $\mathbf{\%}$ \\
\hline Cesário & 563 & 50,67 \\
Vaginal & 531 & 47,79 \\
Aborto & 17 & 1,53 \\
\hline
\end{tabular}

A Tabela 2 mostra as características dos partos normais. Pode-se perceber que houve um número elevado (79) de parturientes que foram submetidas ao método de episiotomia. 
Tabela 2 - Características dos partos normais em parturientes atendidas em um hospital e maternidade da cidade de Sobral/CE

\begin{tabular}{lcc}
\hline Características do parto vaginal & $\mathbf{n}$ & $\mathbf{\%}$ \\
\hline Episiotomia & 79 & 35,42 \\
Episiorrafia & 19 & 8,52 \\
Laceração & 125 & 56,05 \\
\hline
\end{tabular}

A Tabela 3 faz uma correlação entre tipo de parto e índice de Apgar aos 5 minutos, onde nota-se que tanto o parto vaginal quanto o parto do tipo cesário tiveram índice de Apgar aos minutos entre os escores de 8 a 10 em sua maioria.

A Tabela 4 expõe os resultados sociodemográficos e obstétricos (faixa etária, número de consultas pré-natal realizadas, idade gestacional em semanas e graus de escolaridade) das parturientes correlacionado aos tipos de partos. Percebe-se que a maior incidência de cesárea deu-se na faixa etária de 21-30 anos (47,73\%), seguida de 24,95\% da faixa etária de 31-40 e de parto normal deu-se, também, na faixa etária de $21-30$ anos (41,60\%), seguida de $40,11 \%$ da faixa etária de 12-20 anos (Tabela 4).

Tabela 3 - Tipo de parto correlacionado ao Índice de Apgar aos 5 minutos em parturientes atendidas em um hospital e maternidade da cidade de Sobral/CE

\begin{tabular}{|c|c|c|c|c|c|c|}
\hline \multirow{3}{*}{ Tipo de parto } & \multicolumn{6}{|c|}{ Índice de Apgar* } \\
\hline & \multicolumn{2}{|c|}{$0-3$} & \multicolumn{2}{|c|}{$4-7$} & \multicolumn{2}{|c|}{$8-10$} \\
\hline & $\mathbf{n}$ & $\%$ & $\mathbf{n}$ & $\%$ & $\mathbf{n}$ & $\%$ \\
\hline Cesária & 12 & 2,17 & 46 & 8,34 & 493 & 89,47 \\
\hline Vaginal & 06 & 1,14 & 46 & 8,79 & 471 & 90,05 \\
\hline
\end{tabular}

*Média=8,62; Desvio padrão=1.

Quanto ao número de consultas pré-natal correlacionado ao tipo de parto, demonstra-se que tanto nos parto vaginal quanto nos partos do tipo cesário os valores foram semelhantes em mulheres que realizaram de 7 ou mais consultas (Tabela 4).

Quando se compara idade gestacional e tipo de parto, observa-se que houve mais partos em gestantes com idade gestacional entre 37 e 41 anos, e em sua maioria realizaram parto do tipo cesário $(69,52 \%)$ (Tabela 4$)$.

Fazendo um paralelo entre o grau de escolaridade e tipo de parto, há o predomínio de gestantes com o ensino médio e que realizaram o parto do tipo cesário $(43,86 \%)$ e gestantes com o ensino fundamental II que tiveram parto vaginal $(45,54 \%)$ (Tabela 4$)$.

Tabela 4 - Variáveis sociodemográfica e obstétrica correlacionadas aos tipos de parto em parturientes atendidas em um hospital e maternidade da cidade de Sobral/CE

\begin{tabular}{|c|c|c|c|c|}
\hline \multirow{2}{*}{$\begin{array}{l}\text { Variáveis } \\
\text { Faixa etária* }\end{array}$} & \multicolumn{2}{|c|}{ Cesário } & \multicolumn{2}{|c|}{ Vaginal } \\
\hline & $\mathbf{n}$ & $\%$ & $\mathbf{n}$ & $\%$ \\
\hline De $12-20$ & 130 & 23,50 & 215 & 40,11 \\
\hline De $21-30$ & 264 & 47,73 & 223 & 41,60 \\
\hline De 31 a 40 & 138 & 24,95 & 87 & 16,23 \\
\hline De 41 a 50 & 17 & 3,07 & 11 & 2,05 \\
\hline De 51 a 54 & 04 & 0,72 & 0 & 0 \\
\hline \multicolumn{5}{|l|}{$\mathrm{N}^{0}$ de consultas*** } \\
\hline De 1-3 & 34 & 6,09 & 31 & 6,16 \\
\hline De 4-6 & 161 & 28,85 & 158 & 31,41 \\
\hline$\geq 7$ & 363 & 65,05 & 314 & 62,42 \\
\hline \multicolumn{5}{|l|}{ Idade gestacional $* * *$} \\
\hline De $22-27$ & 11 & 2,00 & 07 & 1,52 \\
\hline De $28-36$ & 122 & 22,26 & 103 & 22,44 \\
\hline De $37-41$ & 381 & 69,52 & 345 & 75,16 \\
\hline$\geq 42$ & 34 & 6,20 & 04 & 0,87 \\
\hline \multicolumn{5}{|l|}{ Grau de instrução } \\
\hline Analfabeto & 01 & 0,18 & 01 & 0,18 \\
\hline Fundamental I & 43 & 7,76 & 46 & 8,72 \\
\hline Fundamental II & 200 & 36,10 & 240 & 45,54 \\
\hline Médio & 243 & 43,86 & 206 & 39,08 \\
\hline Superior completo & 33 & 5,95 & 14 & 2,65 \\
\hline Superior incompleto & 34 & 6,13 & 20 & 3,79 \\
\hline
\end{tabular}

*Média=25,79; Desvio padrão=7,09. **Média=7,4; Desvio padrão=2,77. ***Média=37,55; Desvio padrão=2,93. 
A Tabela 5 demonstra a(s) indicação(ões) para a realização de partos do tipo cesário. Nota-se que em sua maioria foi justificado pelo fato de reduzir riscos à vida da parturiente ou ao feto, porém observa-se um percentual de $16,54 \%$ que foram realizadas somente por eletiva (de acordo com o registro no prontuário).

Tabela 5 - Indicação para os partos cesarianos em parturientes atendidas em um hospital e maternidade da cidade de Sobral/CE

\begin{tabular}{lll}
\hline Indicação & N & \% \\
\hline Alto risco/pré-eclâmpsia & 150 & 27,27 \\
Desproporção cefálico/pélvica & 79 & 14,36 \\
Eletiva de alto risco & 58 & 10,54 \\
Eletiva & 91 & 16,54 \\
Sofrimento fetal & 35 & 6,36 \\
Oligohidramnio & 21 & 3,81 \\
Eletiva/pós-datismo & 34 & 4,36 \\
Ruptura prematura & 31 & 5,63 \\
Crescimento excessivo (macrossomia) & 10 & 1,81 \\
Apresentação pélvica & 17 & 3,09 \\
Natimorto & 06 & 1,09 \\
Placenta prévia & 10 & 1,81 \\
Outros & 08 & 1,44
\end{tabular}

Outros: alto risco/eclampsia, hemorragia, anormalidades dos órgãos pélvicos, falha de indução

\section{DISCUSSÃO}

Nas últimas décadas, o Brasil vivenciou uma mudança no padrão de nascimento, em que as operações cesarianas tornaram-se a via de parto mais comum, chegando a $85 \%$ dos partos realizados nos serviços privados de saúde. No sistema público de saúde a taxa é consideravelmente menor, de $40 \%{ }^{12}$. No presente trabalho o parto cesário teve predomínio sobre o parto vaginal (Tabela 1). A Organização Mundial da Saúde (OMS) discorre que não há justificativa para um percentual de partos cesáreos superior a $15 \%$ em nenhuma região do mundo ${ }^{13}$. O Ministério da Saúde do Brasil, igualmente, discorre que elevadas taxas de cesáreas são razões determinantes da morbimortalidade materna e perinatal ${ }^{14}$.

Apesar dos progressos obstétricos e do elevado percentual de partos cesarianas realizados no Brasil, ainda não foram atingidas melhorias na mortalidade perinata ${ }^{15}$. Houve redução nas taxas de mortalidade perinatal sem aumento nas taxas de parto cesarianas e reputam ser mais provável que a redução da taxa de mortalidade perinatal dos partos realizados e supervisionados seja mais uma relação direta da melhoria do atendimento neonatal do que do tipo de parto $^{16}$.

A ascensão na taxa de cesarianas é um fenômeno da obstetrícia mundial, mas no Brasil, líder das estatísticas ${ }^{17,18}$, o tema tem um conceito diferenciado, sendo visto como uma questão que exige enfretamento por todos os envolvidos na atenção à saúde da mulher. Fatores socioculturais e relacionados ao sistema de saúde, além da subvalorização dos riscos associados ao procedimento, são preocupantes. Sabe-se que, quando comparada ao parto normal, a chance de morbidade materna grave se eleva 2 vezes entre mulheres submetidas à cesariana intraparto e 2 a 3 vezes em caso de cesariana eletiva. Esta última situação, além de alongar a permanência hospitalar, associa-se à maior morbidade e mortalidade após alta ${ }^{19}$.

No Brasil, a cada ano, aproximadamente 1,5 milhões de mulheres têm parto vaginal ${ }^{20}$. No presente estudo observou-se que entre os partos vaginais a presença da aplicação da episiotomia foi grande proporção (TABELA 2). A Organização Mundial de Saúde (OMS) sugere uma taxa ideal de episiotomia nos diversos serviços em torno de $10 \%$, realidade em muitos países europeus. Infelizmente, no Brasil, em alguns centros, a situação é ainda adversa, pois o procedimento é realizado em, aproximadamente, 94\% dos partos vaginais ${ }^{21}$.

Para alguns autores a vitalidade do recém-nascido é um fator que pode está relacionado ao tipo de parto. Essa vitalidade é estimada pelo índice de Apgar, no primeiro e quinto minutos de vida, cujos valores apresentando-se baixos indicam menos oportunidade de sobrevivência ${ }^{22}$, indicando a importância da vigilância adequada do trabalho de parto e auxilio adequado logo após o nascimento. No presente estudo foi observado que os índices de Apgar tiveram bons valores tanto no parto cesário quanto no vaginal, mostrando percentuais semelhantes (Tabela 3). O índice de Apgar demonstra as condições de vitalidade do recém-nascido e está diretamente relacionado com a qualidade do atendimento ao parto. Este, quando em deficiência, pode resultar em sofrimento fetal e levar a baixos índices de Apgar, mesmo que o bebê nasça a termo e com peso adequado ${ }^{22}$.

O aumento na incidência da gravidez nos extremos da vida reprodutiva, antes dos 20 e após os 35 anos de idade, é uma realidade. Muitos estudos descrevem o impacto dos extremos da vida reprodutiva, adolescência e idade avançada sobre os resultados maternos e perinatais com conclusões conflitantes. Algumas condições maternas associadas ao possível comprometimento fetal costumam ser mais frequentes e mais graves nesta faixa etária e, portanto, elevam o número de cesáreas ${ }^{23}$. Quando analisando a correlação do tipo de parto com a idade materna, constatou-se que à medida que a idade materna avança, a frequência de partos normais diminui (Tabela 4), corroborando com a literatura.

É indiscutível a importância do pré-natal como item de proteção para a mãe e o bebê. Existe uma forte 
associação entre cuidados pré-natais adequados e melhores resultados perinatais, seja em mulheres adolescentes ou mais maduras ${ }^{24}$. Entre os fatores associados ao aumento na incidência de cesáreas, estaria a ausência de um atendimento pré-natal que prepare a gestante para o parto vaginal e o despreparo psicológico das mulheres, condicionado pelo ambiente social e pelos meios de comunicação, gerando cesáreas a pedido da paciente ${ }^{25}$. De acordo com as informações colhidas, a maioria das gestantes que tiveram parto cesário e normal relataram um número de consultas pré-natal maior ou igual a sete (Tabela 4). Talvez essa discordância esteja relacionada às indicações visualizadas na Tabela 5.

A pouca ou nenhuma orientação durante o pré-natal sobre as reais indicações de cesariana e os benefícios e desvantagens de cada tipo de parto, afiliada ao progresso científico e ao aprimoramento da técnica cirúrgica, caracterizam um modelo de assistência médica fortemente decisiva da opção pelo parto operatório ${ }^{26}$. Muitas vezes é transmitida à gestante, durante o pré-natal, a ideia injustificável de que o parto cesáreo é mais seguro para a mãe e para o bebê e, portanto, uma forma mais "moderna" de parto $^{27}$.

De acordo com dados colhidos, tanto os partos de via vaginal como de cesário, os maiores percentuais estiveram na idade gestacional de 37-41 semanas (Tabela 4). Corroborados em análises científicas, econômicas e fundadas no direito de escolha da mulher ${ }^{28}$, vários países incluindo o Brasil, têm se mobilizado através de seus serviços nacionais de saúde, entidades de classe e movimentos sociais em prol do resgate do nascimento como um processo biológico natural, destinando a cesariana para condições de excepcionalidade, quando justificadas cientificamente $24,29,30$. Por outro lado, algumas interpretações menos elaboradas do ponto de vista científico atribuem ao procedimento cesariano o indutor de resultados maternos e neonatais desfavoráveis, sem levar em conta as indicações do referido procedimento.

Quanto ao tipo de parto correlacionada com o grau de escolaridade, aquelas que detém maior grau, neste grupo de mulheres, há uma maior taxa de parto cesáreo, sugerindo a existência de outras condições além dos clínicos ${ }^{30,31}$. A escolaridade materna está fortemente associada ao tipo de parto quando as mães com maior grau de instrução apresentam uma chance seis vezes maior de terem seus filhos de parto cesário ${ }^{33}$. Esses achados na literatura corroboram com os resultados do presente trabalho, conforme visualizado na Tabela 4.

Em síntese, ao referirem da "cultura da cesariana no Brasil", o parto operatório passou a simbolizar para a parturiente e sua família um atendimento médico diferenciado ("parto sem dor", "ausência de asfixia ao nascimento", "sexualidade, e para o obstetra, maior controle do ato médico e de seu horário de trabalho) ${ }^{34}$.

Com relação as indicações que levaram ao parto cesário, a maioria deles foi indicado com pertinência (Tabela 5) pois essa via deve ser indicada sempre que houver qualquer indício ou ameaça de dano ao binômio materno-perinatal ${ }^{35}$. No entanto foi observado cirurgia por eletiva, ou seja, a cesariana a pedido materno ou sem indicação médica realizada antes de iniciado o trabalho de parto e que é feita na ausência de indicações maternas e fetais $^{35}$. A taxa de cesariana vem crescendo ao longo dos anos e por várias razões as mulheres têm solicitado a via cirúrgica como forma de nascimento. História pessoal ou de pessoas próximas de parto vaginal com desfechos negativos são mencionados pelas mulheres, medo de sentir dor, falta de conhecimento sobre os mecanismos de alívio da dor no trabalho de parto e preocupação com sofrimento fetal e cesariana de urgência são alguns assuntos envolvidos na escolha e desejo pela cesariana programada ${ }^{12}$.

Deve-se ressaltar que, quando realizada sob indicações médicas específicas, a operação cesariana é uma cirurgia essencial para a saúde materna e infantil ${ }^{12}$. Entretanto, pode levar ao aumento do risco de complicações graves quando realizada sem a correta indicação. Diante do uso excessivo de operações cesarianas desnecessárias no contexto nacional, torna-se necessária a qualificação da atenção à gestante, a fim de garantir que a decisão pela via de parto considere os ganhos em saúde e seus possíveis riscos, de forma claramente informada e compartilhada entre a gestante e a equipe de saúde que a atende ${ }^{12}$.

Como limitação do presente estudo, pode-se considerar a impossibilidade de acesso com os profissionais envolvidos, uma vez que se trata de trabalho retrospectivo. Esse acesso permitiria determinar quais protocolos são utilizados para se determinar tais condutas. Outra limitação também está associada a incorreções devido a falhas no preenchimento dos prontuários e à falta de informações ou informações incompletas.

\section{CONCLUSÃO}

As informações obtidas com base nesse estudo das características clínicas e epidemiológicas dos tipos de parto poderão contribuir para as políticas de saúde pública.

Apesar da diferença entre o número de parto cesáreo e normal ter sido pequena, pode-se afirmar que o parto cesário teve um alto índice, visto que ultrapassa a porcentagem máxima destes que é pré-estabelecida pelo Ministério da Saúde. Esse índice elevado pode ser justificado em parte quando se observa uma boa representatividade de mulheres com faixa etária fora dos padrões estabelecidos e os elevado números de casos de complicações obstétricas e/ou fetais, fatos que indicam o parto do tipo cesário. 
Outro ponto que deve ser ressaltado ao observar os resultados obtidos é que houve um alto índice de partos no intervalo de idade entre 12 e 20 anos, significando que há um grande número de jovens dando a luz cedo. Este fato chama a atenção para a necessidade de investir em políticas de educação sexual voltadas para esse público, a fim de controlar a natalidade em idades muito jovens evitando, então, passar pelo parto cesário e ficarem vulneráveis a possíveis complicações em decorrência deste.

Participação dos autores: Moreira AC, Santos VMPR, Andrade SG, realizaram coleta de dados, fizeram compilação dos dados, participaram na escrita do manuscrito. Vasconcelos $Y$, Bem SS, Castro $R Q$, fizeram compilação dos dados, participaram na escrita do manuscrito. Carneiro JKR, ajudou a interpretar os resultados, fez revisão/correção do manuscrito. Oliveira MAS, orientou, ajudou a interpretar os resultados, fez revisão/correção do manuscrito.

\section{REFERÊNCIAS}

1. Barros FC, Bhutta ZA, Batra M, Hansen TN, Victora $\mathrm{CG}$, Rubens CE, et al. Global report on preterm birth and stillbirth (3 of 7): evidence for effectiveness of interventions. BMC Pregnancy Childbirth. 2010;10(Suppl 1):S3. doi: 10.1186/1471-2393-10-S1-S3.

2. Bhutta ZA, Das JK, Bahl R, Lawn JE ,Salam RA, Paul VK, et al. Can available interventions end preventable deaths in mothers, newborn babies, and stillbirths, and at what cost? Lancet. 2014;384(9940):347-70. doi: 10.1016/S01406736(14)60792-3.

3. Brasil. Ministério da Saúde. Secretaria de Atenção à Saúde. Departamento de Ações Programáticas Estratégicas. Pré-natal e puerpério: atenção qualificada e humanizada: manual técnico. Brasília: Ministério da Saúde; 2006. (Série A. Normas e Manuais Técnicos; Série Direitos Sexuais e Direitos Reprodutivos; Caderno no 5). Disponível em: http:// bvsms.saude.gov.br/bvs/publicacoes/manual_pre_natal_ puerperio_3ed.pdf.

4. Andreucci CB, Cecatti JG. Desempenho de indicadores de processo do Programa de Humanização do Pré-natal e Nascimento no Brasil: uma revisão sistemática. Cad Saude Publica. 2011;27(6):1053-64. http://dx.doi.org/10.1590/ S0102-311X2011000600003.

5. Nagahama EEI, Santiago SM. Humanização e equidade na atenção ao parto em município da região Sul do Brasil. Acta Paul Enferm. 2008;21(4):609-15. http://dx.doi.org/10.1590/ S0103-21002008000400012.

6. Muñoz LA, Sanchez X, Arcos E, Vollrath A, Bonatti C. Vivenciando a maternidade em contextos de vulnerabilidade social: uma abordagem compreensiva da fenomenologia social. Rev Latino Am Enferm. 2013;21(4):1-7. Disponível em: http://www.scielo.br/pdf/rlae/v21n4/pt_0104-1169rlae-21-04-0913.pdf.

7. Xavier RB, Jannotti CB, Silva KS, Martins AC. Risco reprodutivo e renda familiar: análise do perfil de gestantes. Cien Saude Colet. 2013;18(4):1161-71. http://dx.doi. org/10.1590/S1413-81232013000400029.

8. Bittencourt F, Vieira JB, Almeida ACCH. Concepção de gestantes sobre o parto cesariano. Cogitare Enferm. 2013;18(3):515-20. http://dx.doi.org/10.5380/ce.v18i3.33565

9. Senna MCM. Equidade e política de saúde: algumas reflexões sobre o Programa Saúde da Família. Cad Saude Publica. 2002;18(Supl.):S203-11. http://dx.doi. org/10.1590/S0102311X2002000700020.
10. Baracat MMZ, Nobre FS. Participação social como elemento de análise da sustentabilidade: estudo do Programa Brasileiro de DST/AIDS. Cad EBAPE. 2013;11(1):41-64. http://dx.doi. org/10.1590/S1679-39512013000100005.

11. Santa Casa de Misericórdia de Sobral. Apresentações [citado mar.2018]. Disponível em: http://stacasa.com.br/site/ apresentacoes/.

12. Brasil. Ministério da Saúde. Comissão Nacional de Incorporação de Tecnologias no SUS (CONITEC). Diretrizes de atenção à gestante: a operação cesariana. Relatório de recomendações. Brasília: Ministério da Saúde; 2015. p.5. Disponível em: http://conitec.gov.br/images/Consultas/ Relatorios/2015/Relatorio_PCDTCesariana_CP.pdf.

13. Appropriate technology for birth. Lancet 1985;326:436-7. https://doi.org/10.1016/S0140-6736(85)92750-3.

14. Brasil. Ministério da Saúde. Parto, aborto e puerpério, assistência humanizada à mulher. Brasília: Ministério da Saúde; 2001. Disponível em: http://bvsms.saude.gov.br/ bvs/publicacoes/cd04_13.pdf.

15. De Lorenzi DRS, Tanaka, ACA, Bozzetti MC, Ribas FE, Weissheimer L. A natimortalidade como indicador de saúde perinatal. Cad Saúde Pública. 2001;17(1):141-6. http://dx.doi. org/10.1590/S0102-311X2001000100014.

16. Duarte G, Coltro PS, Bedone, RV, NogueiraAA, Gelonezzi GM, Franco LJ. Tendência das formas de resolução da gravidez e sua influência sobre as taxas de mortalidade perinatal. Rev Saúde Pública. 2004;38(3):379-84. http:// dx.doi.org/10.1590/S0034-89102004000300007.

17. Rattner D, Rabello Neto DL, Lansky S, Vilela ME, Bastos MH. As cesarianas no Brasil: situação no ano de 2010, tendências e perspectivas. In: Brasil. Ministério da Saúde. Secretaria de Vigilância em Saúde. Departamento de Análise de Situação de Saúde. Saúde Brasil 2011: uma análise da situação de saúde e a vigilância da saúde da mulher. Brasília (DF): Ministério da Saúde; 2012.p.371-97. Disponível em: http://bvsms.saude.gov. br/bvs/publicacoes/saude_brasil_2014_analise_situacao.pdf.

18. Rede Interagencial de Informação para a Saúde (RIPSA). Indicadores básicos para a saúde no Brasil: conceitos e aplicações. 2a ed. Brasília (DF): Organização Pan-Americana da Saúde; 2008. Disponível em: http://tabnet.datasus.gov.br/ tabdata/livroidb/2ed/indicadores.pdf.

19. Villar J, Carroli G, Zavaleta N, Donner A, Wojdyla D, Faundes A, et al. Maternal and neonatal individual risks 
and benefits associated with caesarean delivery: multicentre prospective study. BMJ. 2007;335(7628):1025. doi: https:// doi.org/10.1136/bmj.39363.706956.55.

20. Brasil. Ministério da Saúde. DATASUS. Nascidos vivos. Brasil. Nascimento por residência da mãe segundo Região. Tipo de parto: vaginal, 2007 [citado jun.2017]. Disponível em: http://tabnet.datasus.gov.br/cgi/tabcgi.exe?sinasc/cnv/ nvuf.def.

21. Carvalho CCMC, Souza ASR, Moraes Filho, OB. Episiotomia seletiva: avanços baseados em evidências. Femina. 2010;38(5). Disponível em: http://files.bvs.br/upload/S/0100-7254/2010/ v38n5/a008.pdf.

22. Magalhães MC, Carvalho MS. Atenção hospitalar perinatal e mortalidade neonatal no município de Juiz de Fora, Minas Gerais. Rev Bras Saúde Matern Infant. 2003;3(3):329-37. http://dx.doi.org/10.1590/S1519-38292003000300012.

23. Santos GHN, Martins MG, Sousa MS, Batalha SJC. Impacto da idade materna sobre os resultados perinatais e via de parto. Rev Bras Ginecol Obstet. 2009;31(7):326-34. http://dx.doi. org/10.1590/S0100-72032009000700002.

24. Gama SGN, Szwarcwald CL, Leal MC, Theme Filha MM. Gravidez na adolescência como fator de risco para baixo peso ao nascer no município do Rio de Janeiro, 1996 a 1998. Rev Saúde Pública. 2001;35:74-80. http://dx.doi.org/10.1590/ S0034-89102001000100011.

25. Lamy GO, Moreno BS. Assistência pré-natal e preparo para o parto. Omnia Saúde. 2013;10(2):19-35.

26. Fabri RH, Murta EFC. Tipos de parto e formas de assistência médica em Uberaba-MG. Rev Bras Ginecol Obstet. 1999;21:99-104. http://dx.doi.org/10.1590/S010072031999000200007.

27. Barbosa GP, Giffin K, Angulo-Tuesta A, Gama AS, Chor
D, D’Orsi E, et al. Parto cesáreo: quem o deseja? Em quais circunstâncias? Cad Saúde Pública. 2003;19:1611-34. http:// dx.doi.org/10.1590/S0102-311X2003000600006.

28. Ventura M. Direitos reprodutivos no Brasil. São Paulo: M. Ventura; 2002.

29. Reynolds A, Ayres-de-Campos D, Costa MA, Santos C, Campos I, Montenegro N. Influência de três medidas organizativas na redução da taxa de cesarianas de um Hospital de Apoio Perinatal Diferenciado. Acta Med Port. 2004;17(3):193-8.

30. Chaillet N, Dubé E, Dugas M, Francoeur D, Dubé J, Gagnon S, et al. Identifying barriers and facilitators towards implementing guidelines to reduce caesarean section rates in Quebec. Bull World Health Organ. 2007;85(10):791-7. doi: 10.2471/BLT.06.039289.

31. Gould JB, Davey B, Stafford RS. Socioeconomic differences in rates of cesarean section. New Engl J Med. 1989;321:233-9. doi: 10.1056/NEJM198907273210406.

32. Nascimento LFC. Grau de instrução materna: um marcador obstétrico. In: V Congresso Brasileiro de Saúde Coletiva, Resumos. Águas de Lindóia: ABRASCO; 1997. p.271.

33. Haidar FH, Oliveira UF, Nascimento LFC. Escolaridade materna: correlação com os indicadores obstétricos. Cad Saúde Pública. 2001;17(4):1025-9. http://dx.doi.org/10.1590/ S0102-311X2001000400037.

34. Freitas PF, Drachler ML, Leite JCC, Grassi PR. Desigualdade social nas taxas de cesariana em primíparas no Rio Grande do Sul. Rev Saúde Pública 2005; 39:761-7. http://dx.doi. org/10.1590/S0034-89102005000500010.

35. Câmara R, Burlá M, Ferrari J, Lima L, Amim Júnior J, Braga A, Rezende Filho. Cesariana a pedido materno. Rev Col Bras Cir. 2016;43(4):301-10. doi: 10.1590/0100-69912016004002.

Recebido:28 ago. 2017.

Aceito: 20 dez. 2018. 